
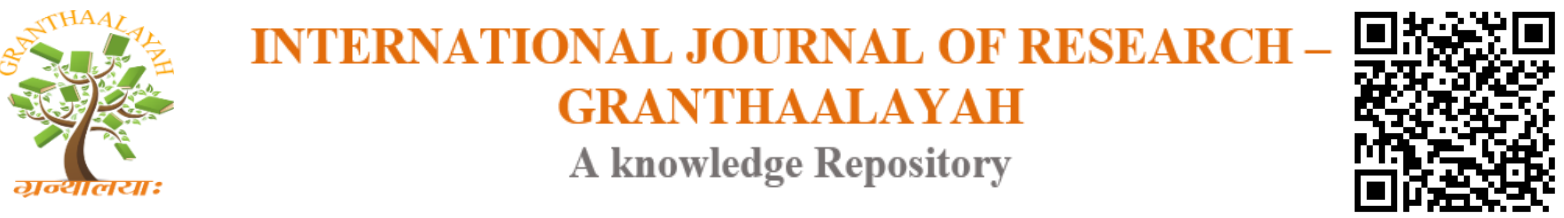

Science

\title{
MHD DOUBLE DIFFUSIVE AND CHEMICALLY REACTIVE FLUID FLOW THROUGH A ROTATING POROUS PLATE
}

\author{
M.Umamaheswar ${ }^{1}$, M.C.Raju ${ }^{* 2}$, S.V. K. $\operatorname{Varma}^{3}$, C. Sucharitha ${ }^{3}$ \\ 1 , *2 Department of Mathematics, Annamacharya Institute of Technology and Sciences \\ (Autonomous), Rajampet, Cuddapah District, A.P., India \\ ${ }^{3}$ Department of Mathematics, S. V. University, Tirupathi, India
}

\begin{abstract}
In this paper MHD rotating double diffusive and chemically reactive fluid flow past a vertical porous plate with thermal radiation and heat absorption/generation is studied. The nondimensional governing equations involved in the present analysis are solved by using finite difference technique. The effects of various physical parameters on velocity, temperature and concentration along with skin friction, the rate of heat transfer in the form of Nusselt number and the rate of mass transfer in the form of Sherwood number are studied through the graphs and tables.
\end{abstract}

Keywords: Double Diffuse and Chemically Reaction; MHD; Mass Transfer; Thermal Radiation; Porous Plate; Heat Absorption.

Cite This Article: M.Umamaheswar, M.C.Raju, S.V. K. Varma, and C. Sucharitha. (2017). "MHD DOUBLE DIFFUSIVE AND CHEMICALLY REACTIVE FLUID FLOW THROUGH A ROTATING POROUS PLATE." International Journal of Research - Granthaalayah, 5(7), 363-373. https://doi.org/10.29121/granthaalayah.v5.i7.2017.2143.

\section{Introduction}

Simultaneous heat and mass transfer from different geometries embedded in porous medium has many Engineering and Geophysical applications such as geothermal reservoirs, drying of porous solids, thermal insulation, enhanced oil recovery, packed bed catalytic reactors, cooling of nuclear reactors, and underground energy transport. The science of magneto hydrodynamics (MHD) was concerned with geophysical and astrophysical problem for a number of years. In recent years, the possible use of MHD is to affect a flow stream of an electrically conducting fluid for the purpose of thermal protection, braking, propulsion and control. From the point of applications, model studies on the effect of magnetic field on the convection flow have been made by several investigators. From technological point of view, MHD convection flow problems are also very significant in the field of stellar and planetary magnetospheres, aeronautics, chemical engineering and electronics. Model studies of the above phenomena of MHD convection have been made by many. Ahmed et al. [1] considered Hall Effect on transient 
MHD flow past an impulsively started vertical plate in a porous medium with ramped temperature, rotating and heat absorption. Bharat et al. [2] discussed the effects of mass transfer on MHD free convective radiation flow over an impulsively started vertical plate embedded in a porous medium. Ravi Kumar et al. [3] considered MHD double diffusive and chemically reactive flow through porous medium bounded by two vertical plates. Harinath Reddy et al. [4] discussed unsteady MHD free convection flow of a kuvshinski fluid past a vertical porous plate in the presence of chemical reaction and heat source/sink. Raju et al. [5] studied MHD convective flow through porous medium in a horizontal channel with insulated and impermeable bottom wall in the presence of viscous dissipation and joule heating. Mamtha et al. [6] considered thermal diffusion effect on MHD mixed convection unsteady flow of a micro polar fluid past a semi-infinite vertical porous plate with radiation and mass transfer. Das et al. [7] discussed mass transfer effects on MHD flow and heat transfer past a vertical porous plate through a porous medium under oscillatory suction and heat source. Ananda Reddy et al. [8] considered thermo diffusion and chemical effects with simultaneous thermal and mass diffusion in MHD mixed convection flow with ohmic heating. Pal et al. [9] studied double diffusive magneto hydrodynamic heat and mass transfer of nanofluids over a nonlinear stretching/shrinking sheet with viscous-Ohmic dissipation and thermal radiation. Ravi kumar et al. [10] discussed thermal diffusive free convective radiating flow over an impulsively started vertical porous plate in conducting field. Ibrahim and Shankar [11] considered MHD boundary layer flow and heat transfer of nanofluids past a permeable stretching sheet with velocity, thermal and solutal slip boundary conditions. Abdul-Kahar et al. [12] discussed scaling group transformation for boundary-layer flow of a nanofluid past a porous vertical stretching surface in the presence of chemical reaction with heat radiation.EugenMagyari and AsteriosPantokratoras [13] discussed note on the effect of thermal radiation in the linearized Roseland approximation on the heat transfer characteristics of various boundary layer flows. Chien-Hsin [14] discussed magneto hydrodynamic mixed convection of a power-law fluid past a stretching surface in the presence of thermal radiation and internal heat generation/absorption. Elbashbeshy et al. [15] studied effects of thermal radiation and magnetic field on unsteady mixed convection flow and heat transfer over an exponentially stretching surface with suction in the presence of internal heat generation/absorption. Abdul Gaffra et al. [16] considered Thermal radiation and heat generation/ absorption effects on viscoelastic double-diffusive convection from an isothermal sphere in porous media. Chamkha [17] discussed Effects of heat absorption and thermal radiation on heat transfer in a fluid-particle flow past a surface in the presence of a gravity field. Khali et al. [18] studied mixed convection flow in lid-driven enclosure filled with a fluid-saturated porous medium. Ziya et al. [19] discussed influence of thermal radiation and heat generation/absorption on MHD heat transfer flow of a micro polar fluid past a wedge with hall and ion slip currents. Ravi Kumar et al. [20] studied combined effects of heat absorption and MHD on convective Rivlin-Ericksen flow past a semi-infinite vertical porous plate with variable temperature and suction. Vinayaka Prasad et al. [21] discussed influence of international heat generation/absorption, thermal radiation, magnetic field, variable fluid property and viscous dissipation on heat transfer characteristics of a Maxwell fluid over a stretching sheet.

\section{Mathematical Formulation}

We consider a viscous incompressible, electrically conducting, heat absorbing/generating and chemically reacting Newtonian fluid flow past an infinite vertical porous. A magnetic field of 
uniform strength is applied perpendicular to the plate. Let $\mathrm{x}^{*}$-axis is taken along the plate in the vertically upward direction and the $\mathrm{y}^{*}$-axis is taken perpendicular to the plate. The fluid as well as the plate is in a state of rigid body rotation with a uniform angular velocity $\Omega$ about $y$-axis. At time $t \leq 0$, the plate is maintained at the temperature higher than ambient temperature $\mathrm{T}_{\infty}$ and the fluid is at rest. At time $t>0$, the plate is linearly accelerated with increasing time in its own plane and also At time $t^{*}>0$ the temperature and Concentration of the plate $y^{*}=0$ is raised exponentially with time $t$ and thereafter remains constant and that of $y^{*} \rightarrow \infty$ is lowered to end. It is assumed that the effect of viscous dissipation is negligible. Based on the above assumptions, the equations governing the flow along with the relevant boundary conditions are given below:

$$
\begin{aligned}
& \frac{\partial u^{*}}{\partial t^{*}}+2 \Omega V^{*}=v \frac{\partial^{2} u^{*}}{\partial y^{* 2}}+g \beta\left(T^{*}-T_{\infty}^{*}\right)+g \beta^{*}\left(C^{*}-C_{\infty}^{*}\right)-\frac{\sigma B_{0}^{2} u^{*}}{\rho}-\frac{v}{K^{*}} u^{*} \\
& \frac{\partial V^{*}}{\partial t^{*}}-2 \Omega u^{*}=v \frac{\partial^{2} V^{*}}{\partial y^{* 2}}-\frac{\sigma B_{0}^{2} V^{*}}{\rho}-\frac{v}{K^{*}} V^{*} \\
& \rho C_{p} \frac{\partial T^{*}}{\partial t^{*}}=k \frac{\partial^{2} T^{*}}{\partial y^{* 2}}+Q^{*}\left(T^{*}-T_{\infty}^{*}\right)-\frac{\partial q_{r}^{*}}{\partial y^{*}} \\
& \frac{\partial C^{*}}{\partial t^{*}}=D \frac{\partial^{2} C^{*}}{\partial y^{* 2}}-K r^{*}\left(C^{*}-C_{\infty}^{*}\right)
\end{aligned}
$$

The corresponding initial and boundary conditions are

$$
\left.\begin{array}{rl}
u^{*}=0, V^{*} & =0, T^{*}=T_{\infty}, \mathrm{C}^{*}=C_{\infty} \quad \text { for all } y^{*}, t^{*} \leq 0 \\
t^{*}>0: u^{*} & =U_{0} a^{*} t^{*}, V^{*}=U_{0} a^{*} t^{*}, \mathrm{~T}^{*}=T_{\infty}^{*}+\left(T_{w}^{*}-T_{\infty}^{*}\right) e^{a^{*} t^{*}}, \mathrm{C}^{*}=C_{\infty}^{*}+\left(C_{w}^{*}-C_{\infty}^{*}\right) e^{a^{*} t^{*}} \text { at } y^{*}=0 \\
u^{*} & \rightarrow 0, T^{*} \rightarrow T_{\infty}^{*}, \mathrm{C}^{*} \rightarrow C_{\infty}^{*} \quad \text { as } y^{*} \rightarrow \infty
\end{array}\right\}
$$

The non-dimensional quantities are as follows:

$$
\begin{aligned}
& u=\frac{u^{*}}{U_{0}}, V=\frac{V^{*}}{U_{0}}, t=\frac{t^{*} U_{0}^{2}}{v}, y=\frac{y^{*} U_{0}}{v}, \theta=\frac{T^{*}-T_{\infty}^{*}}{T_{w}^{*}-T_{\infty}^{*}}, \mathrm{C}=\frac{C^{*}-C_{\infty}^{*}}{C_{w}^{*}-C_{\infty}^{*}}, a=\frac{a^{*} v}{U_{0}^{2}}, \frac{\partial q_{r}^{*}}{\partial y^{*}}=4\left(T^{*}-T_{\infty}\right) I^{*}, \\
& G r=\frac{v g \beta\left(T_{w}^{*}-T_{\infty}^{*}\right)}{U_{0}^{3}}, \text { (Grashof number) }
\end{aligned}
$$

$G m=\frac{v g \beta^{*}\left(C_{w}^{*}-C_{\infty}^{*}\right)}{U_{0}^{3}}$, (Modified Grashof number)

$M=\frac{\sigma B_{0}^{2} v}{\rho U_{0}^{2}}$, (Magnetic parameter)

$k_{1}^{2}=\frac{\Omega v}{U_{0}^{2}},($ Rotation parameter $)$

$K=\frac{k U_{0}^{2}}{v^{2}},($ Permeability of the porous medium)

$\operatorname{Pr}=\frac{\rho v C_{p}}{k},($ Prandtl number $)$

$Q=\frac{Q^{*} v^{2}}{\rho C_{p} U_{0}^{2}},($ Heat generation/absorption $)$ 
$R=\frac{4 \nu I^{*}}{\rho C_{p} U_{0}^{2}}$, (Radiation parameter)

$S c=\frac{v}{D},($ Schmidt number $)$

$K r=\frac{K r^{*} v}{U_{0}^{2}}$ (Chemical reaction parameter)

Introducing the non-dimensional quantities the equations (1)-(3) reduces to following form

$\frac{\partial u}{\partial t}+2 k_{1}^{2} V=\frac{\partial^{2} u}{\partial y^{2}}+G r \theta+G m C-M u-\frac{1}{K} u$

$\frac{\partial V}{\partial t}-2 k_{1}^{2} u=\frac{\partial^{2} V}{\partial y^{2}}-M V-\frac{1}{K} V$

$\frac{\partial \theta}{\partial t}=\frac{1}{\operatorname{Pr}} \frac{\partial^{2} \theta}{\partial y^{2}}+Q \theta-R \theta$

$\frac{\partial C}{\partial t}=\frac{1}{S c} \frac{\partial^{2} C}{\partial y^{2}}-K r C$

The corresponding initial and boundary conditions are

$u=0, V=0, \theta=0, \quad \mathrm{C}=0 \quad$ for all $\quad \mathrm{y}, \mathrm{t} \leq 0$

$t>0: u=a t, V=a t, \theta=e^{a t}, \mathrm{C}=e^{a t}$ at $y=0$

$u \rightarrow 0, V \rightarrow 0, \theta \rightarrow 0, \quad \mathrm{C} \rightarrow 0 \quad$ as $y \rightarrow \infty$

\section{Method of Solution}

Equations (6)-(9) are linear partial differential equations and are to be solved with the initial and boundary conditions (10). In fact the exact solution is not possible for this set of equations and hence we solve these equations by finite-difference method. The equivalent finite difference schemes of equations for (6)-(9) are as follows:

$$
\begin{aligned}
& \frac{u_{i, j+1}-u_{i, j}}{\Delta t}+k_{1}^{2} V_{i, j}=G r \theta_{i, j}+G m C_{i, j}+\frac{u_{i-1, j}-2 u_{i, j}+u_{i+1, j}}{(\Delta y)^{2}}-M u_{i, j}-\frac{1}{K} u_{i, j} \\
& \frac{V_{i, j+1}-V_{i, j}}{\Delta t}-2 k_{1}^{2} u_{i, j}=\frac{V_{i-1, j}-2 V_{i, j}+V_{i+1, j}}{(\Delta y)^{2}}-M V_{i, j}-\frac{1}{K} V_{i, j} \\
& \frac{\theta_{i, j+1}-\theta_{i, j}}{\Delta t}=\frac{1}{\operatorname{Pr}} \frac{\theta_{i-1, j}-2 \theta_{i, j}+\theta_{i+1, j}}{(\Delta y)^{2}}+Q \theta_{i, j}-F \theta_{i, j} \\
& \frac{C_{i, j+1}-C_{i, j}}{\Delta t}=\frac{1}{S c} \frac{C_{i-1, j}-2 C_{i, j}+C_{i+1, j}}{(\Delta y)^{2}}-K r C_{i, j}
\end{aligned}
$$

Here, the suffix $i$ refer to $y$ and $\mathrm{j}$ to time. The mesh system is divided by taking $\Delta y=0.1$. From the initial condition in (10), we have the following equivalent:

$$
u(i, 0)=0, \theta(i, 0)=0, C(i, 0)=0 \text { for all } i
$$


The boundary conditions from (10) are expressed in finite-difference form as follows

$$
\begin{aligned}
& u(0, j)=a t, v(0, j)=a t, \theta(0, j)=e^{a t}, C(0, j)=e^{a t} \text { for all } j \\
& u\left(i_{\max }, j\right)=0, v\left(i_{\max }, j\right)=0, \theta\left(i_{\max }, j\right)=0, C\left(i_{\max }, j\right)=0 \text { for all } j
\end{aligned}
$$

(Here $\mathrm{i}_{\max }$ was taken as 200)

The primary velocity at the end of time step viz, $u(i, j+1)(i=1,200)$ is computed from (11) andthe secondary velocity at the end of time step viz, $\mathrm{V}(\mathrm{i}, \mathrm{j}+1)(\mathrm{i}=1,200)$ is computed from (12) in terms of velocity, temperature and concentration at points on the earlier time-step. After that $\theta(i, j+1)$ is computed from (13) and then $\mathrm{C}(\mathrm{i}, \mathrm{j}+1)$ is computed from (14). The procedure is repeated until $\mathrm{t}=0.5$ (i.e. $\mathrm{j}=500$ ). During computation $\Delta \mathrm{t}$ was chosen as 0.001 .

\section{Skin-friction:}

The skin-friction in non-dimensional form is given by the relation

$$
\tau=\left(\frac{d u}{d y}\right)_{y=0} \text {, where } \tau=\frac{\tau^{1}}{\rho U_{0}^{2}}
$$

\section{Rate of heat transfer:}

The dimensionless rate of heat transfer in terms of Nusselt number is given by

$$
N u=-\left(\frac{d \theta}{d y}\right)_{y=0}
$$

\section{Rate of mass transfer:}

The dimensionless rate of mass transfer in terms of Sherwood number is given by

$$
S h=-\left(\frac{d C}{d y}\right)_{y=0}
$$

\section{Results and Discussion}

In order to look into the physical insight of the problem, the expressions obtained in previous section are studied with help of graphs from figures 1-13. The effects of various physical parameters viz., the Schmidt number (Sc), the thermal Grashof number (Gr), the mass Grashof number $(\mathrm{Gm})$, magnetic parameter $(\mathrm{M})$, rotation parameter and chemical reaction parameter $(\mathrm{Kr})$ etc; are studied numerically by choosing arbitrary values. In fig.1, effect of thermal Grashof number on primary velocity is presented. As Gr increases, primary velocity also increases. This is due to the buoyancy which is acting on the fluid particles due to gravitational force that enhances the fluid primary velocity.A similar effect is noticed from Fig.2, in the presence of modified Grashof number, which also increases fluid primary velocity. In figure 3, velocity profiles are displayed with the variation in magnetic parameter. From this figure it is noticed that velocity gets reduced by the increase of magnetic parameter. Because the magnetic force which is applied perpendicular to the plate, retards the flow, which is known as Lorentz force.Hence the presence of this retarding force reduces the fluid velocity. Figure 4 exhibits the primary velocity profiles for various values of $\mathrm{K}$. From this figure it is observed that primary velocity increases with an increase in Permeability parameter. Figure 5, depicts the rotation parameter effect on 
primary velocity, as rotation parameter increases, primary velocity also increases.In figure 6, secondary velocity profiles are displayed with the variation in magnetic parameter. From this figure it is noticed that secondary velocity gets reduced by the increase of magnetic parameter.Figure 7 exhibits the secondary velocity profiles for various values of K. From this figure it is observed that secondary velocity increases with an increase in Permeability parameter. Figure 8, depicts the rotation parameter effect on secondary velocity, as rotation parameter increases, primary secondary velocity also increases.Effect of $\operatorname{Pr}, \mathrm{Q}$ and $\mathrm{R}$ on temperature are studied in figures 9, 10 and 11. From these figures it is noticed that temperature decreases as $\mathrm{Pr}$ and $\mathrm{R}$ increases and temperature increases as heat source increases. Effect of heat source parameter on temperature is studied in figure 11. From this figure, it is noticed that temperature increases as heat source parameter increases. Influence of Schmidt number on concentration is shown in figure 12, from this figure it is noticed that concentration decreases with an increase in Schmidt number. Because, Schmidt number is a dimensionless number defined as the ratio of momentum diffusivity and mass diffusivity, and is used to characterize fluid flows in which there are simultaneous momentum and mass diffusion convection processes. Therefore concentration boundary layer decreases with an increase in Schmidt number.From figure 13, we observe that the Concentration $(\mathrm{C})$ decreases as chemical reaction $(\mathrm{Kr})$ increases. The effect of various physical parameters on Skin friction is presented in table 1. From this table it is noticed that Skin friction decreases with an increase in Grashof number, modified Grashof number, permeability parameter, rotation parameter of secondary velocity whereas skin friction increases with an increase in magnetic parameter and rotation parameter of secondary velocity. The effect of various physical parameters on Nusselt number are presented in table 2. From this table it is noticed that Nusselt number increases with an increase in Prandtl number and radiation parameter whereas Nusselt number decreases with an increase in heat source parameter.The effect of various physical parameters on Sherwood number are presented in table 3. From this table it is noticed that Sherwood number increase with increasing values of Schmidt number and chemical parameter.

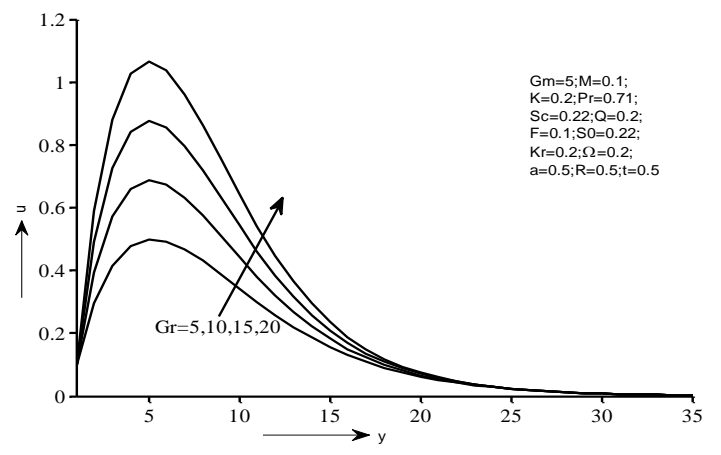

Figure 1: Effect of Grashof number on Primary velocity

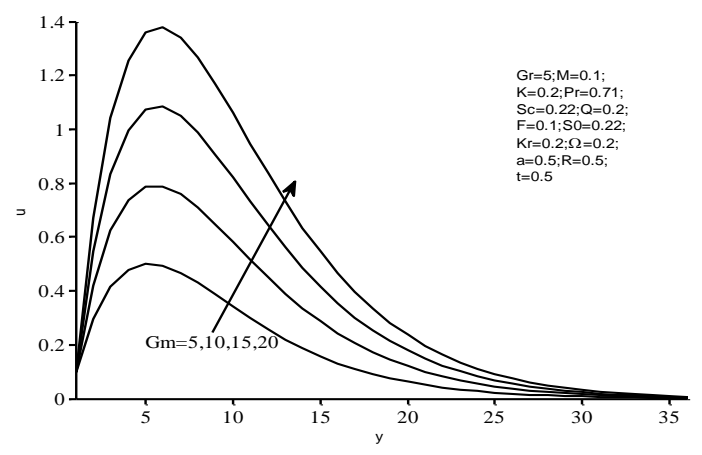

Figure 2: Effect of modified Grashof number on Primary velocity 


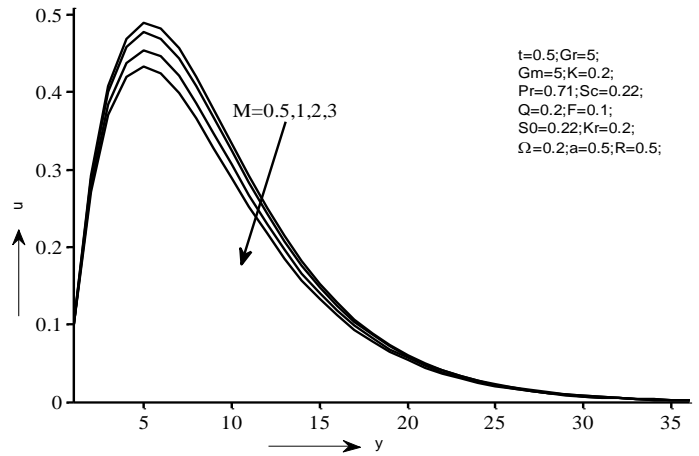

Figure 3: Effect of Magnetic parameter on Primary velocity

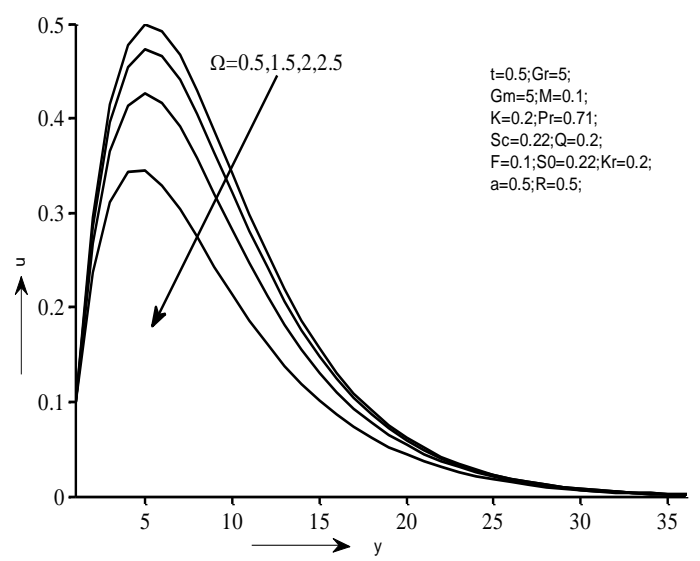

Figure 5: Effect of rotation parameter on Primary velocity

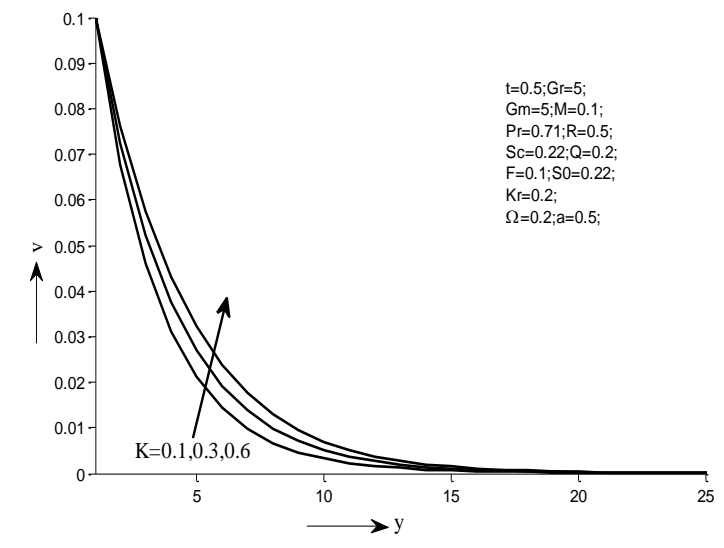

Figure 7: Effect of Permeability parameter on Secondary velocity

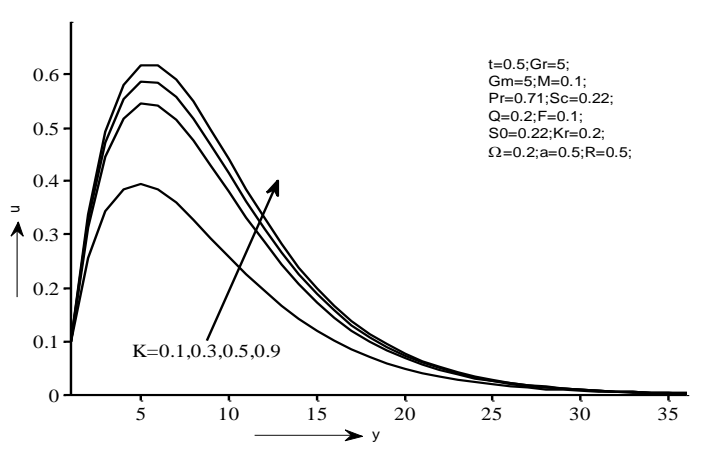

Figure 4: Effect of Permeability parameter on Primary velocity

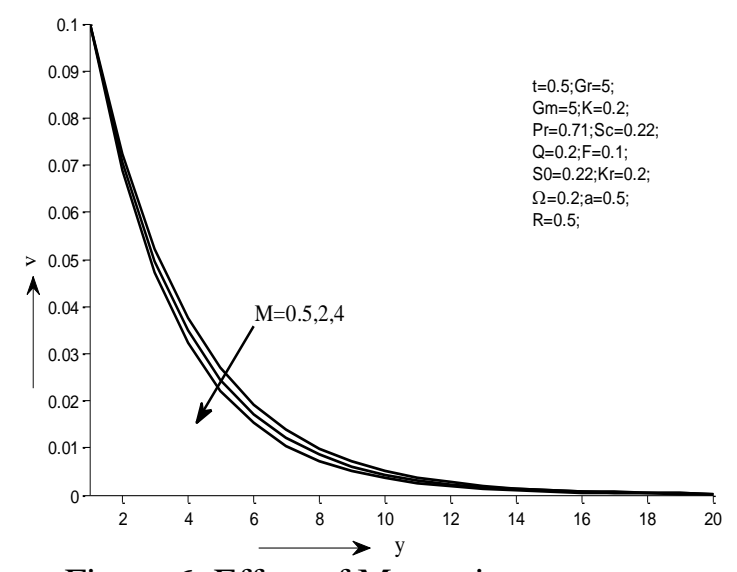

Figure 6: Effect of Magnetic parameter on Secondary velocity

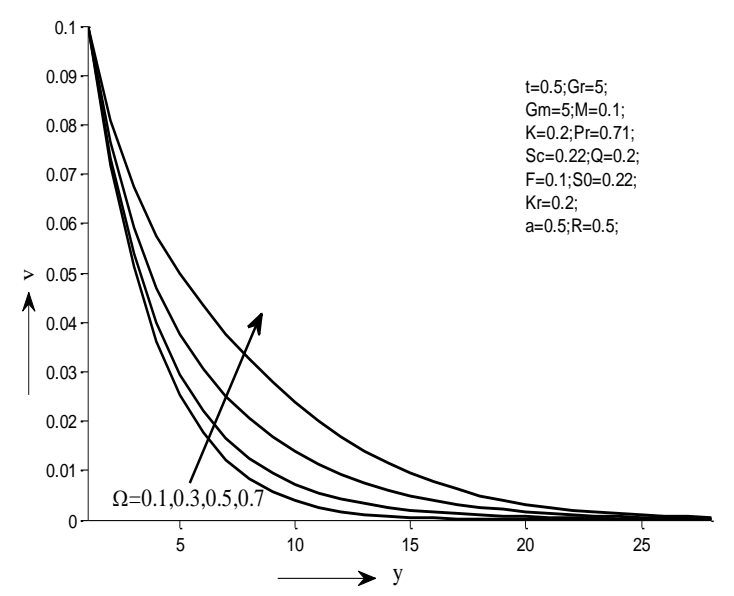

Figure 8: Effect of rotation parameter on Secondary velocity 


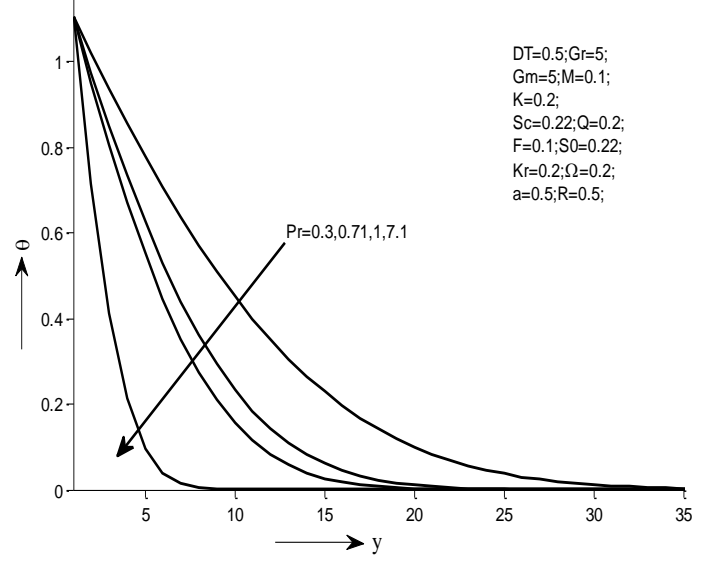

Figure 9: Effect of Prandtl number on temperature

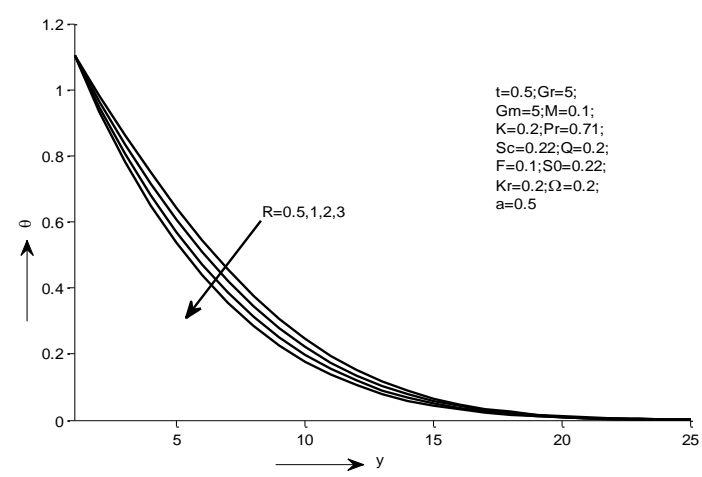

Figure 11: Effect of Radiation parameter on temperature

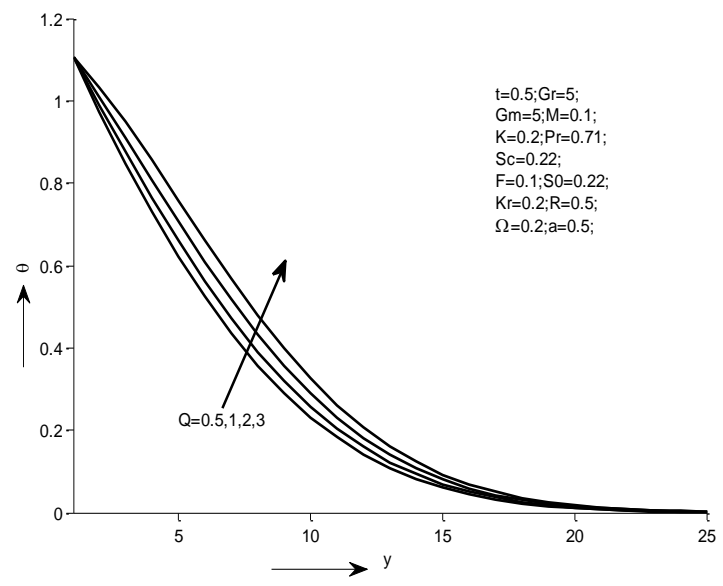

Figure 10: Effect of heat source on temperature

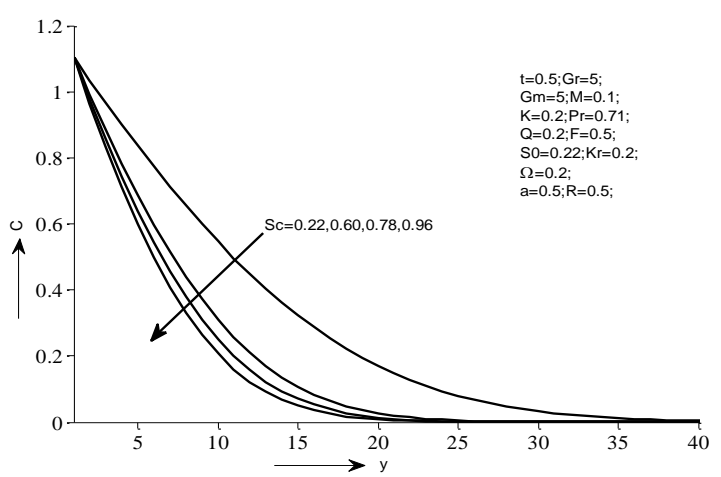

Figure 12: Effect of Schmidt number on Concentration

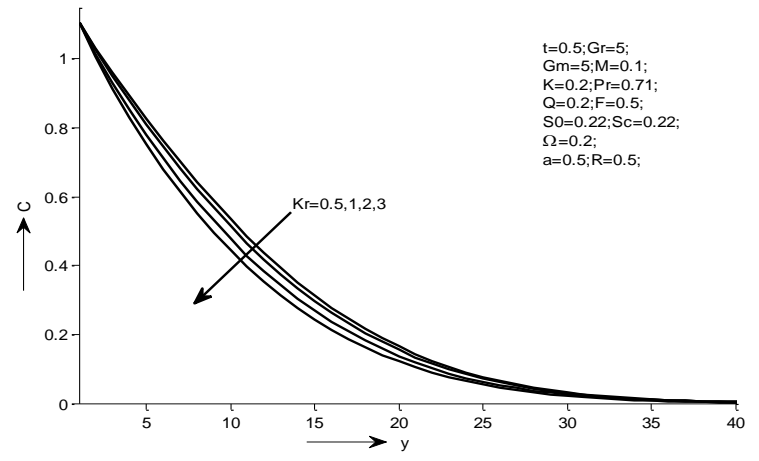

Figure 13: Effect of chemical reaction parameter on Concentration

Table1: Variation in skin friction under the influence of Grashof number, modified Grashof number, magnetic parameter, porosity parameter and rotation parameter

\begin{tabular}{|l|l|l|l|l|l|l|}
\hline $\mathbf{G r}$ & $\mathbf{G m}$ & $\mathbf{M}$ & $\mathbf{K}$ & $\Omega$ & $\boldsymbol{\tau}_{\mathbf{x}}$ & $\boldsymbol{\tau}_{\mathbf{y}}$ \\
\hline 5 & 5 & 3 & 1.2 & 2.2 & 5.8445 & 0.0089 \\
\hline 10 & 5 & 3 & 1.2 & 2.2 & 5.5072 & 0.0067 \\
\hline 15 & 5 & 3 & 1.2 & 2.2 & 5.2635 & 0.0054 \\
\hline 20 & 5 & 3 & 1.2 & 2.2 & 5.0324 & 0.0024 \\
\hline 5 & 10 & 3 & 1.2 & 2.2 & 5.6356 & 0.2337 \\
\hline 5 & 15 & 3 & 1.2 & 2.2 & 5.4309 & 0.1868 \\
\hline
\end{tabular}




\begin{tabular}{|l|l|l|l|l|l|l|}
\hline 5 & 20 & 3 & 1.2 & 2.2 & 5.3235 & 0.0536 \\
\hline 5 & 5 & 0.5 & 1.2 & 2.2 & 2.2790 & 0.0232 \\
\hline 5 & 5 & 1 & 1.2 & 2.2 & 3.9619 & 0.0258 \\
\hline 5 & 5 & 2 & 1.2 & 2.2 & 4.7387 & 0.0325 \\
\hline 5 & 5 & 3 & 2.2 & 2.2 & 3.3392 & 0.0028 \\
\hline 5 & 5 & 3 & 3.2 & 2.2 & 3.1400 & 0.0019 \\
\hline 5 & 5 & 3 & 4.2 & 2.2 & 3.0403 & 0.0012 \\
\hline 5 & 5 & 3 & 1.2 & 3.2 & 3.1142 & 0.0019 \\
\hline 5 & 5 & 3 & 1.2 & 4.2 & 3.1254 & 0.0017 \\
\hline 5 & 5 & 3 & 1.2 & 8.2 & 3.1365 & 0.0014 \\
\hline
\end{tabular}

Table 2: Variations in skin friction and Nusselt number under the influence of Prandtl number, heat source parameter and radiation parameter

\begin{tabular}{|l|l|l|l|l|l|}
\hline $\mathbf{P r}$ & $\mathbf{Q}$ & $\mathbf{R}$ & $\boldsymbol{\tau} \mathbf{x}$ & $\boldsymbol{\tau} \mathbf{N}$ & $\mathbf{N u}$ \\
\hline 0.71 & 1 & 0.22 & 2.2324 & 0.0121 & 2.4282 \\
\hline 1 & 1 & 0.22 & 1.6543 & 0.0326 & 4.4974 \\
\hline 3 & 1 & 0.22 & 1.5464 & 0.0462 & 5.4583 \\
\hline 7.1 & 1 & 0.22 & 1.3542 & 0.0571 & 6.5026 \\
\hline 2 & 3 & 0.22 & 2.5832 & 0.0028 & 1.9562 \\
\hline 2 & 4 & 0.22 & 2.3826 & 0.0045 & 2.1823 \\
\hline 2 & 1 & 0.5 & 2.8319 & 0.1595 & 1.0485 \\
\hline 2 & 1 & 1 & 2.7950 & 0.1356 & 1.0492 \\
\hline 2 & 1 & 2 & 2.7366 & 0.1252 & 1.0498 \\
\hline 2 & 1 & 0.22 & 2.9820 & 0.0767 & 1.2566 \\
\hline 2 & 1 & 0.22 & 3.2820 & 0.3413 & 1.3472 \\
\hline 2 & 1 & 0.22 & 3.7820 & 0.8238 & 1.5468 \\
\hline
\end{tabular}

Table 3: Effect of chemical reaction parameter and Schmidt number on skin friction and Sherwood number

\begin{tabular}{|l|l|l|l|l|}
\hline $\mathbf{K r}$ & $\mathbf{S c}$ & $\boldsymbol{\tau \mathbf { x }}$ & $\boldsymbol{\tau \mathbf { y }}$ & $\mathbf{S h}$ \\
\hline 3 & 1 & 2.9356 & 0.0010 & 0.8081 \\
\hline 5 & 1 & 2.8762 & 0.0207 & 1.2738 \\
\hline 6 & 1 & 2.8560 & 0.0960 & 1.3198 \\
\hline 3 & 1 & 3.0213 & 0.1330 & 0.1903 \\
\hline 3 & 2 & 3.0649 & 0.0026 & 0.4004 \\
\hline 3 & 3 & 3.1086 & 0.0022 & 0.9918 \\
\hline
\end{tabular}

\section{Conclusions}

In the analysis of the flow the following significant conclusions are made:

- The primary velocity of the fluid increases when the values of rotation parameter increased and the secondary velocity decreases in the same case.

- The temperature of the fluid enhances for increasing values of heat source parameter. 
- The existence of chemical reaction leads to decrease the concentration of the fluid.

\section{References}

[1] N. Ahmed and K.Kr. Das, "Hall effect on transient MHD flow past an impulsively started vertical plate in a porous medium with ramped temperature, rotating and heat absorption", Applied Mathematical Sciences, (2013), 7(51), 2525-2535.

[2] K S Bharat and S Nityananda, "The effects of mass transfer on MHD free convective radiation flow over an impulsively started vertical plate embedded in a porous medium", Journal of Applied analysis and Computation, (2015), 5, 18-27.

[3] V. Ravikumar, M. C. Raju, G.S.S. Raju and A. L Chamkha, "MHD double diffusive and chemically reactive flow through porous medium bounded by two vertical plate", International Journal of Energy \& Technology, (2013), 5: 01-08.

[4] S. Harinath Reddy, M.C. Raju, K. Reddy, "Unsteady MHD free convection flow of a kuvshinski fluid past a vertical porous plate in the presence of chemical reaction and heat source/sink", International Journal of Engineering Research in Africa, (2015), 14, 13-27.

[5] K.V.S Raju, T. S. Reddy , M. C. Raju, P.V. SatyaNarayana and S.V. Ramana "MHD convective flow through porous medium in a horizontal channel with insulated and impermeable bottom wall in the presence of viscous dissipation and joule heating", Ain Shams Eng J, (2013), $5,543-551$.

[6] B. Mamtha, M. C. Raju, S.V.K. Varma, "Thermal diffusion effect on MHD mixed convection unsteady flow of a micro polar fluid past a semi-infinite vertical porous plate with radiation and mass transfer", International Journal of Engineering research in Africa, (2015), 13, 21-37.

[7] S.S. Das, A. Satapathy, J.K. Das, and J.P. Panda, "Mass transfer effects on MHD flow and heat transfer past a vertical porous plate through a porous medium under oscillatory suction and heat source”, International Journal of Heat and Mass Transfer, (2009), 52(25-26), 5962-5969.

[8] N. Ananda Reddy, S.V.K. Varma, M.C. Raju , "Thermo diffusion and chemical effects with simultaneous thermal and mass diffusion in MHD mixed convection flow with ohmic heating", $\mathrm{J}$ Naval Architect Mar Eng, (2009), 6, 84-93.

[9] D. Pal and M. Gopinath, "Double diffusive magneto hydrodynamic heat and mass transfer of nanofluids over a nonlinear stretching/shrinking sheet with viscous-Ohmic dissipation and thermal radiation", Propulsion and Power Research, (2017), 6(1), 58-69.

[10] V. Ravi kumar, M. C. Raju, G.s.S. Raju and S.V.K. Varma, "Thermal diffusive free convective radiating flow over an impulsively started vertical porous plate in conducting field", Journal of Physical Mathematics, (2016), 7(1), 1-8.

[11] W. Ibrahim and B. Shankar, "MHD boundary layer flow and heat transfer of a nanofluids past a permeable stretching sheet with velocity, thermal and solutal slip boundary conditions", Computers and fluids, (2013), 75(20), 1-10.

[12] R. Abdul-Kahar, R. Kandasamy and Muhaimin, "Scaling group transformation for boundarylayer flow of a nanofluids past a porous vertical stretching surface in the presence of chemical reaction with heat radiation", computers and Fluids, (2011), 52(30), 15-21.

[13] EugenMagyari and AsteriosPantokratoras, "Note on the effect of thermal radiation in the linearized Roseland approximation on the heat transfer characteristics of various boundary layer flows", International Communications in Heat and Mass Transfer, (2011), 38(5), 554-556.

[14] C. Chien-Hsin, "Magnetohydrodynamic mixed convection of a power-law fluid past a stretching surface in the presence of thermal radiation and internal heat generation/absorption", Int J NonLinear Mech, (2009), 44, 596-603.

[15] E.M.A. Elbashbeshy, T.G. Eman and K. M Abdelgaber, "Effects of thermal radiation and magnetic field on unsteady mixed convection flow and heat transfer over an exponentially 
stretching surface with suction in the presence of internal heat generation/absorption", J Egypt Math Soc , (2012), 20, 215-222.

[16] S. Abdul Gaffra, V. Ramachandra Prasad, E. Keshava Reddy and O. Anwar Beg, "Thermal radiation and heat generation/ absorption effects on viscoelastic double-diffusive convection from an isothermal sphere in porous media", Ain Shams engineering Journal, (2015), 6(3), 1009-1030.

[17] Ali J. Chamkha, "Effects of heat absorption and thermal radiation on heat transfer in a fluidparticle flow past a surface in the presence of a gravity field", International Journal of thermal sciences, (2000), 39(5), 605-615.

[18] Khali M. Khanafer and Ali J.Chamkha, "Mixed convection flow in lid-driven enclosure filled with a fluid-saturated porous medium", international Journal of Heat and Mass Transfer, (1999), 42(13), 2465-2481.

[19] U. Ziya, Manojkumar and S. Harmand, "Influence of thermal radiation and heat generation //absorption on MHD heat transfer flow of a micro polar fluid past a wedge with hall and ion slip currents", Thermal Science, (2014), 18(2), S489-S502.

[20] V. Ravikumar, M.C. Raju and G.S.S. Raju, "Combined effects of heat absorption and MHD on convective Rivlin-Ericksen flow past a semi-infinite vertical porous plate with variable temperature and suction", Ain Shams Engineering Journal, (2014), 5, 867-875.

[21] K. Vinayaka Prasad, K. Vairavelu and A. Sujatha, "Influence of international heat generation/absorption, thermal radiation, magnetic field, variable fluid property and viscous dissipation on heat transfer characteristics of a Maxwell fluid over a stretching sheet", Journal of Applied Mechanics, (2013), 6(2), 249-256.

*Corresponding author.

E-mail address: mcrmaths@ yahoo.co.in 